Check for updates

Cite this: RSC Adv., 2017, 7, 49947

Received 3rd October 2017

Accepted 11th October 2017

DOI: $10.1039 / \mathrm{c} 7 \mathrm{ra10912a}$

rsc.li/rsc-advances

\section{Renewable metal-organic-frameworks-coated 3D printing film for removal of malachite green $\dagger$}

\author{
Zhennan Shi,,$^{\mathrm{ab}}$ Chen Xu, tab Fei Chen, ${ }^{\mathrm{ab}}$ Yingxi Wang, ${ }^{\mathrm{ab}}$ Ling Li, (D) *ab Qingtao Meng ${ }^{\mathrm{c}}$ \\ and Run Zhang (D)*cd
}

A renewable metal-organic-frameworks (MOFs)-polylactic acid (PLA) film was designed and prepared in order to remove malachite green (MG) in waste water. The PLA frame was prepared by threedimensional (3D) printing technology, followed by in situ step-by-step growth of Cu-MOFs on its surface. The printed PLA skeleton and the prepared Cu-MOFs/PLA films were characterized by scanning electron microscopy and X-ray powder diffraction. The adsorption capacity of Cu-MOFs/PLA films towards MG was then investigated. Results showed that more than $90 \%$ removal efficiency was observed in adsorbing MG of different concentrations for $10 \mathrm{~min}$. Practical applications of Cu-MOFs/PLA films for removal of MG in waste-water samples were then demonstrated. The used Cu-MOFs/PLA films were found to be recyclable over more than five times after simple washing with acetone. In addition, it was noted that the PLA skeleton could be recovered for the further growth of Cu-MOFs on its surface. The successful development of this technique provides a new strategy for the design of renewable materials for the adsorption of organic dyes in waste water, which holds enormous potential for broad applications in waste-water treatment.

\section{Introduction}

Organic compounds, such as dyes, have been widely used in the dyestuff and pigment industry as well as the food and textile industries. ${ }^{1-6}$ Although dyes play important roles in our daily life, the discharge of large quantities of these toxic organic compounds into the environment causes considerable pollution, and even serious health issues. ${ }^{7-10}$ For example, malachite green (MG) has been widely used in fish breeding and the textile industry; however, the discharge of MG into water causes toxicity to living organisms. ${ }^{11,12}$ To solve these environmental problems, huge efforts have been devoted by researchers and waste treatment plants and industries. However, development of highly efficient and economic approaches for the removal of organic dyes from the environment, particularly in water

${ }^{a}$ Hubei Collaborative Innovation Center for Advanced Organic Chemical Materials, Hubei University, Wuhan 430062, People's Republic of China. E-mail: waitingll@ yahoo.com

${ }^{b}$ Ministry-of-Education Key Laboratory for the Synthesis and Application of Organic Function Molecules, Hubei University, Wuhan City, Hubei Province 430062, People's Republic of China

'School of Chemical Engineering, University of Science and Technology Liaoning, Anshan, 114044, China. E-mail: r.zhang@uq.edu.au

${ }^{d}$ Australian Institute for Bioengineering and Nanotechnology, The University of Queensland, Brisbane, 4072, Australia

$\dagger$ Electronic supplementary information (ESI) available: Supplementary data of SEM images of PLA skeletons, Cu-MOFs, and Cu-MOFs/PLA films. See DOI: 10.1039/c7ra10912a

$\ddagger$ These authors contributed equally to this work. solutions, remains challenging due to their complex aromatic molecular structures. ${ }^{13,14}$

In the past few decades, a variety of methods, such as photodegradation, electrochemical processes, precipitation, adsorption, and ion exchange have been studied for waste-water treatment. ${ }^{15-22}$ Of all these methods, the adsorption approach using suitable adsorbents has emerged as one of the most promising technologies for the removal of organic compounds (e.g. dyes) in waste water. ${ }^{23}$ Among various adsorbents, metalorganic frameworks (MOFs) with unique properties, such as adjustable pore size and high surface area, have drawn increasing interest in the past few decades. ${ }^{24-27} \mathrm{Cu}-\mathrm{MOF}$ is composed of oxygen, nitrogen, other organic ligands and copper ions from the self-assembly of the coordination polymer. In the preparation of $\mathrm{Cu}-\mathrm{MOF}$, the arrangement of organic ligands and metal ions exhibits a clear direction, and different pore structures can be formed with different adsorption properties, optical properties, and electromagnetic properties. As a result, Cu-MOFs were found to have a high adsorption capacity for different organic dyes, thus becoming one of the most widely studied candidates for adsorbents in waste-water treatment. ${ }^{28-32}$

Considering the need for more sustainable and environmentally friendly production materials, some polymers have been introduced to improve the stability of MOFs in water solution and the performance in terms of organic compounds adsorption..$^{33}$ Although a high efficiency of MOFs was observed for the adsorption of organic dyes, recycling of these materials 
administered in water samples is extremely difficult. ${ }^{34}$ To tackle this issue, processes such as centrifugation and filtration have to be carried out to collect the dye-MOFs complexes. ${ }^{35,36}$ Therefore, it is highly desirable if the MOFs can be integrated with a solid skeleton, which will facilitate administration during the adsorption and recovery. ${ }^{37-40}$

Polylactic acid (PLA), obtained from polymerization of a lactic acid derivative, presents as a desirable green polymer for the stabilization of MOFs. ${ }^{\mathbf{4 1 - 4 3}}$ More important, PLA is a biodegradable thermoplastic aliphatic polyester, allowing PLA film to be easily prepared through advanced 3D printing technology ${ }^{\mathbf{4 4 , 4 5}}$ In addition, the existing carbonyl group and carboxyl group can serve as an efficient binding site for the $\mathrm{Cu}$ ions of $\mathrm{Cu}-\mathrm{MOFs}$ through the coordination process, producing $\mathrm{Cu}$ MOFs/PLA films. ${ }^{46}$ This process not only improves the stability of the Cu-MOFs/PLA films, but also facilitates the recycling procedure, avoiding the time-consuming processes of separation and recovery for MOFs adsorbents.

In this contribution, we describe a new approach for the design and preparation of Cu-MOFs/PLA film for the removal organic dyes in waste water. This Cu-MOFs/PLA film was easily produced by the in situ stepwise growing of porous MOFs on a three-dimensional (3D) printed PLA skeleton (Scheme 1). The adsorption properties of the Cu-MOFs/PLA film were then investigated in terms of removing $\mathrm{MG}$ in water. Furthermore, the practical application of these prepared Cu-MOFs/PLA films for removal of MG in real waste-water samples were explored. The designed film was found to be recyclable, allowing its potential in future industrial applications in water treatment.

\section{Experimental section}

\subsection{Materials and physical methods}

1,3,5-Benzenetricarboxylic acid $\left(\mathrm{H}_{3} \mathrm{BTC}\right)$, triethylamine (TEA), PLA, MG, and $\mathrm{Cu}\left(\mathrm{NO}_{3}\right)_{2}$ were purchased from Sinopharm Chemical Reagent Co., Ltd. (Shanghai, China). Reagents and solvents were of analytical reagent grade and were used without further purification, unless otherwise noted. Fresh doubledistilled water was used throughout the experiment.

PLA films were produced using a Z-603S 3D printer (Shenzhen Aurora Technology Co., Ltd). Powder X-ray diffraction (XRD) measurements were performed using a D8 Advance X-ray diffractometer (Bruker Company, USA). Scanning electron microscope (SEM) images were obtained using a JSM6510LV SME (JEOL, Japan). UV-vis absorption spectra were measured using a Lambda 900 UV/VIS/NIR spectrophotometer (PerkinElmer, USA).

\subsection{Preparation of Cu-MOFs/PLA films}

For printing of PLA films, the extrusion temperature of the $3 \mathrm{D}$ printer was set at $210^{\circ} \mathrm{C}$, and the filamentous PLA polymer was printed to form PLA films. The Cu-MOFs/PLA was coated under natural conditions (Fig. 1a). Typically, PLA film was firstly treated with $80 \mathrm{~mL}$ ethanol-water solution $(1: 1, \mathrm{v} / \mathrm{v})$ for 3 hours. This PLA film was then placed into an ethanol-water solution $(80 \mathrm{~mL}, 1: 1, \mathrm{v} / \mathrm{v})$ containing $1.32 \mathrm{~g}$ of $\mathrm{H}_{3} \mathrm{BTC}$ and $0.25 \mathrm{~mL}$ of TEA for $5 \mathrm{~h}$. Further treatment was conducted by placing the film into $80 \mathrm{~mL}$ of ethanol-water solution $(1: 1, \mathrm{v} / \mathrm{v})$ containing $2.22 \mathrm{~g}$ of $\mathrm{Cu}\left(\mathrm{NO}_{3}\right)_{2} \cdot 3 \mathrm{H}_{2} \mathrm{O}$. The film was washed with ethanol and deionized water alternately. Multilayer Cu-MOFscoated PLA films were prepared by repeating the process above for 1 to 8 cycles. Cu-MOFs were prepared as the control group following a reported method..$^{47}$

\subsection{Adsorption and recovery of Cu-MOFs/PLA films}

Fresh MG solutions ( $5 \mathrm{mg} \mathrm{L}^{-1}$ and $10 \mathrm{mg} \mathrm{L}^{-1}$ ) were prepared by dissolving the MG in double-distilled water. For evaluation of

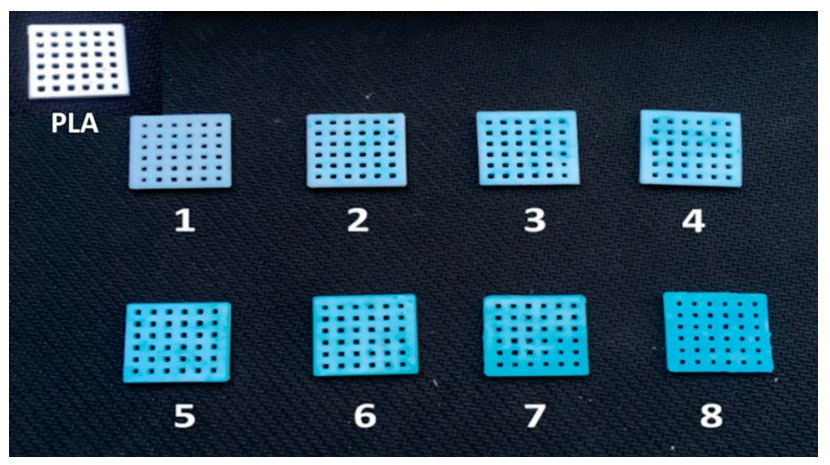

Fig. 1 Images of the Cu-MOFs/PLA- $x(x=1-8)$ films with different layers of $\mathrm{Cu}-\mathrm{MOFs}$ coating.

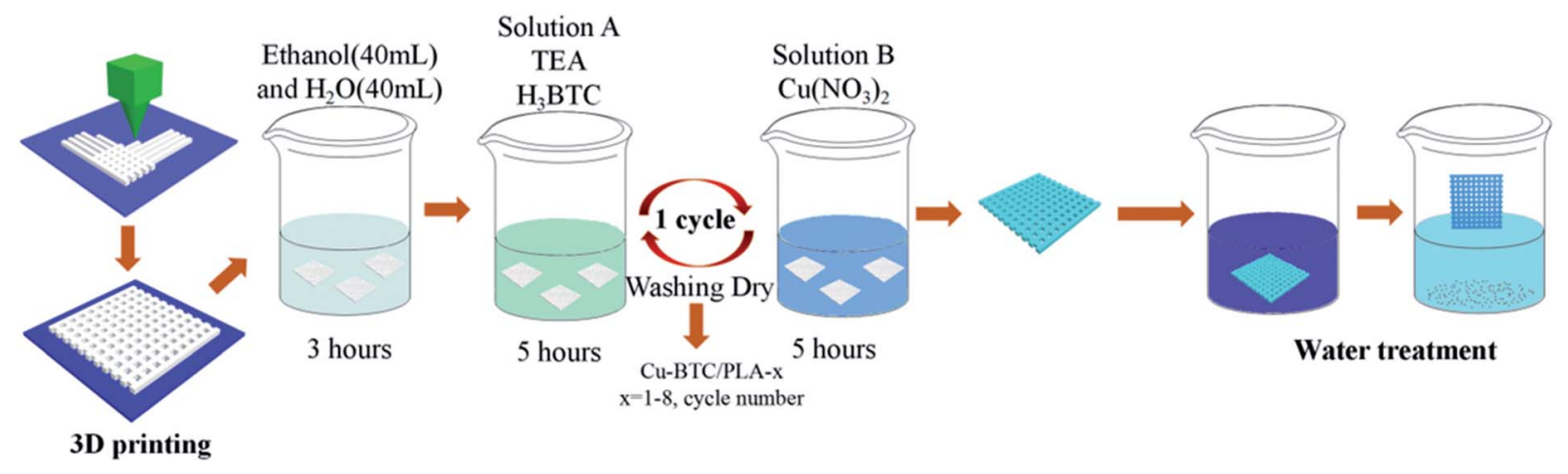

Scheme 1 Schematic illustration of the synthesis of Cu-MOFs/PLA film (1-8 layers) for MG adsorption. 
the adsorption properties, the Cu-MOFs/PLA-8 film was immersed into the solution at both concentrations for $10 \mathrm{~min}$. The film was removed from the solution before measuring the UV-vis spectra of the pre-treated and treated water samples. The control group was created by placing the PLA film in MG-spiked water solution.

For the recovery experiments, the used Cu-MOFs/PLA-8 films were immersed into acetone for $0.5 \mathrm{~h}$. The acetone was replaced with fresh acetone several times during the desorption process. After air drying the recycled Cu-MOFs/PLA-8 films for 2 min, the removal efficiency of these films for MG adsorption was then evaluated following the aforementioned procedures. The adsorption and recovery experiments were repeated five times. Then, the Cu-MOFs/PLA-8 films were immersed into a $1 \mathrm{M} \mathrm{HCl}$ solution for $1 \mathrm{~min}$, and then repeated five times before further growing of Cu-MOFs.

\subsection{Removal of MG from real water sample}

Real water samples were collected from Shahu Lake and Yangtze River in Wuhan, China. The water samples were first filtered to remove solid particles, sand, and organisms. MG was added into the filtrate at a concentration of $10 \mathrm{mg} \mathrm{L}^{-1}$, and the UV-vis spectra of the water samples were measured. The Cu-MOFs/ PLA-8 films were then immersed into the samples $(10 \mathrm{~mL})$ for $10 \mathrm{~min}$. The Cu-MOFs/PLA-8 films were removed, and the UVvis spectra of the treated water samples were then measured for determination of removal efficiency.

\section{Results and discussion}

\subsection{Preparation and characterization of Cu-MOFs/PLA film}

As shown in Scheme 1, the PLA films were printed using a 3D printer. Cu-MOFs were coated on the printed PLA films by a stepwise process, then repeated over 1-8 cycles for the preparation of multilayer Cu-MOFs/PLA- $x(x=1-8)$. It is noted that no stirring or heating process is required for the coating of $\mathrm{Cu}$ MOFs to PLA films, which can significantly reduce the cost for future applications in the waste-water treatment industry. Fig. 1 represents images of $\mathrm{Cu}-\mathrm{MOF}$ /PLA films with different $\mathrm{Cu}$ MOFs wrapping layers. PLA skeletons displayed a white color before coating with Cu-MOFs. As a result of Cu-MOFs coating, the color of the PLA skeleton changed from pure white to blue, and the blue color of the films became darker with increasing number of MOFs layers (1-8 layers).

To study the surface morphology, the PLA skeleton and CuMOFs/PLA- $x(x=1-8)$ films were then characterized by SEM. As shown in Fig. S1, $\dagger$ 3D-printed PLA films showed a smooth surface under SEM. Cu-MOFs were also prepared as the control using the reported method, ${ }^{47}$ and characterized by SEM. As shown in Fig. $\mathrm{S} 2, \uparrow$ the crystals of Cu-MOFs are octahedral with a smooth surface, which is consistent with the reported study. ${ }^{47}$ After in situ stepwise coating, clear changes in PLA skeletons were observed (Fig. S3a and $\mathrm{b} \dagger$ ). The Cu-MOFs were found on the PLA skeleton (Fig. S3c and $d \dagger$ ), suggesting successful growth of Cu-MOFs on PLA by the in situ stepwise process. The $\mathrm{Cu}-$ MOFs/PLA films with various numbers of layers of MOFs

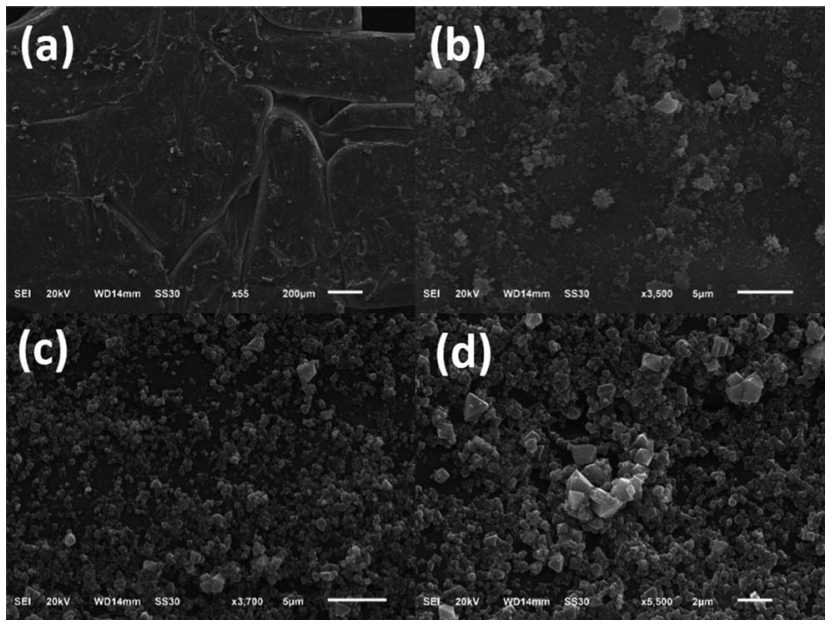

Fig. 2 SEM images of Cu-MOFs/PLA-2 (a), Cu-MOFs/PLA-4 (b), CuMOFs/PLA-6 (c), and Cu-MOFs/PLA-8 (d).

coating $(2,4,6,8)$ are shown in Fig. 2. The amount of $\mathrm{Cu}-\mathrm{MOF}$ coated on the surface of PLA visibly increased with more repeating cycles, confirming that $\mathrm{Cu}$-MOFs can be efficiently grown on the surface of PLA by this stepwise method.

Further evidence of the in situ Cu-MOFs growth was demonstrated by powder XRD measurements. The XRD patterns of the $\mathrm{Cu}-\mathrm{MOFs}$, PLA and Cu-MOFs/PLA with different coating layers $(2,4,6$, and 8$)$ are shown in Fig. 3. No significant changes in the diffraction peaks at $21.2^{\circ}$ and $27.4^{\circ}$ in the XRD pattern were observed, indicating that the PLA films were not affected during Cu-MOFs growth. The diffraction peaks at $21.2^{\circ}$ and $27.4^{\circ}$, attributed to the diffraction of $\mathrm{Cu}-\mathrm{MOFs}$, enhanced with the increasing cycles of MOFs coating. The result further confirms that Cu-MOFs were successfully coated on the PLA films.

\subsection{Adsorption properties}

The adsorption properties of as-prepared Cu-MOFs/PLA films were then investigated by immersing these films into water solutions with different concentrations of MG. Time-dependent adsorption properties of Cu-MOFs/PLA was initially studied by placing the Cu-MOFs/PLA-8 (eight layers of Cu-MOFs) films into

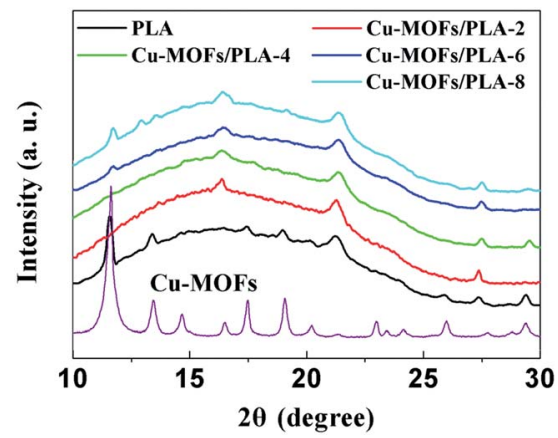

Fig. 3 XRD patterns of PLA, Cu-MOFs, Cu-MOFs/PLA-2, Cu-MOFs/ PLA-4, Cu-MOFs/PLA-6, and Cu-MOFs/PLA-8. 
(a)

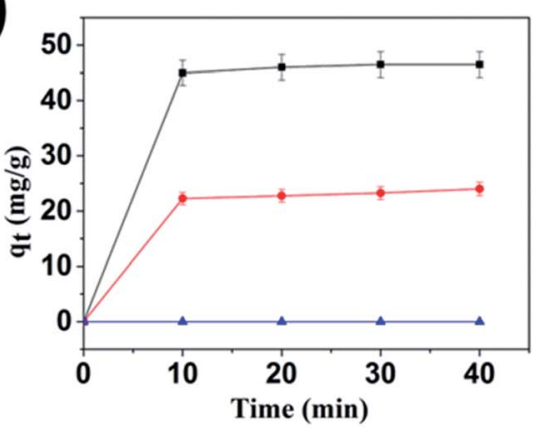

(b)

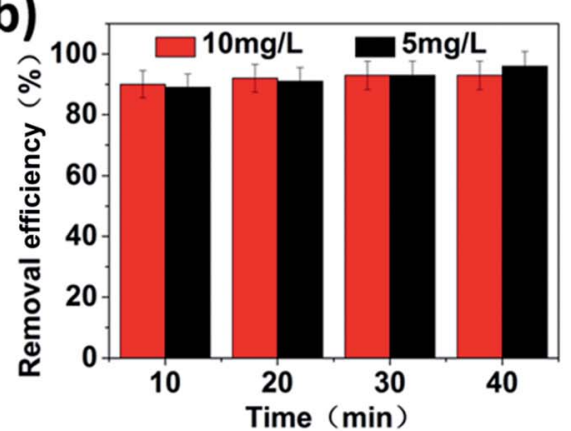

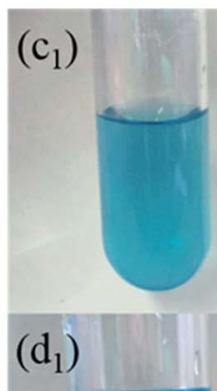

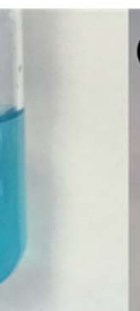

$\left(c_{2}\right)$

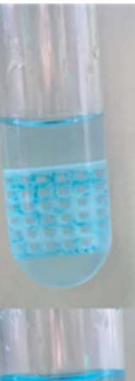

$\left(d_{2}\right)$

$\left(d_{3}\right)$

$\left(c_{3}\right)$

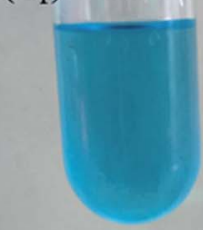

$\left(\mathrm{e}_{1}\right)$

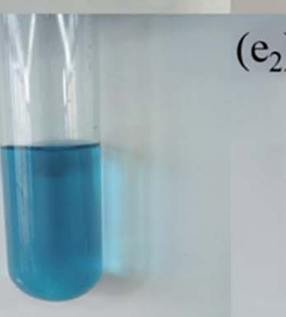

$\left(\mathrm{e}_{2}\right)$

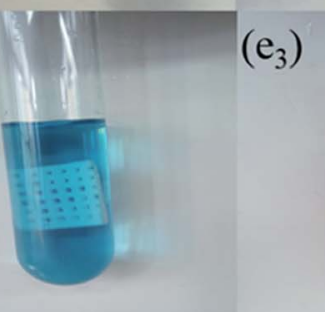

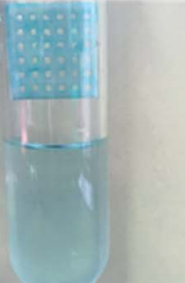

3.
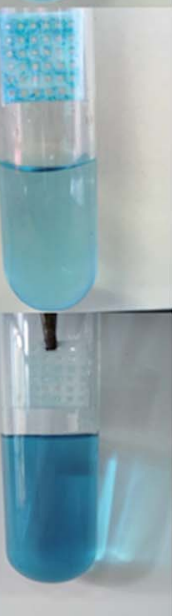

Fig. 4 Time-dependent adsorption capacity (a) and removal efficiency (b) of Cu-MOFs/PLA-8 film towards MG in water solution at the concentrations of $5 \mathrm{mg} \mathrm{L}^{-1}$ and $10 \mathrm{mg} \mathrm{L}^{-1}$. Images of $M G$ water solutions ( $5 \mathrm{mg} \mathrm{L}^{-1}$ for $\mathrm{c}$, and $10 \mathrm{mg} \mathrm{L}^{-1}$ for $\mathrm{d}$, e) before and post-treatment with Cu-MOFs/PLA-8 film (c, d) and PLA film only (e).

an MG water solution ( $5 \mathrm{mg} \mathrm{L}^{-1}$ and $10 \mathrm{mg} \mathrm{L}^{-1}$ ). As shown in Fig. $4 \mathrm{a}$, the maximum adsorption capacity of Cu-MOFs/PLA-8 was obtained after 10 min immersion in MG solution at both concentrations. The removal efficiency for MG was determined to be over $90 \%$ in the initial $10 \mathrm{~min}$ for both concentrations. In contrast, no adsorption of MG was detected for the control group with PLA films only. After treatment with PLA films for $40 \mathrm{~min}$, the removal efficiency was measured to be $0 \%$. These results indicated that the proposed $\mathrm{Cu}-\mathrm{MOF} / \mathrm{PLA}$ films can be used as the absorbent for rapid, high-efficiency, and convenient adsorption of MG in waste water. Removal of MG was also observed by imaging of MG solution pre-/post-treatment with Cu-MOFs/PLA films (Fig. 4c-e). The color of the MG solutions remarkably decreased after treatment with Cu-MOFs/PLA-8 for 10 min (Fig. 4c and d), while the color of PLA film treated group remained almost unaltered (Fig. 4e).

\subsection{Recovery of Cu-MOFs/PLA films and regeneration of PLA}

Desorption experiments were further conducted to test the recyclability of the Cu-MOFs/PLA films. The used Cu-MOFs/PLA films were repeatedly treated with acetone for $30 \mathrm{~min}$. The films were then naturally dried and used as the absorbent for the removal of MG. As shown in Fig. 5, the removal efficiency for MG was maintained at over $80 \%$ after three uses. Even after five uses, the adsorption efficiency was found to be over $60 \%$. The decrease of the adsorption efficiency of Cu-MOFs/PLA films can be attributed to the chemical adsorption of MG by Cu-MOFs, which can be hardly purified by acetone treatment.
Nevertheless, the results demonstrated that the Cu-MOFs/PLA films can be reused at least five times.

Next, we tested the recyclability of the PLA film by placing the used Cu-MOFs/PLA-8 films in $1 \mathrm{M} \mathrm{HCl}$ aqueous solution. It was found that the color of the PLA skeletons changed to pure white after $20 \mathrm{~s}$ treatment (Fig. 6), suggesting that the coated Cu-MOFs particles could be removed from the PLA skeletons. Recovery of PLA skeletons was also confirmed by SEM imaging (Fig. 6e). The smooth surface of white PLA skeletons was observed after $\mathrm{HCl}$ treatment and washing. The results demonstrate that the PLA films are renewable, allowing further coating with Cu-MOFs for MG removal.

The regeneration of Cu-MOFs/PLA films was then demonstrated by in situ stepwise growth methods. As shown in Fig. $6 \mathrm{f}$

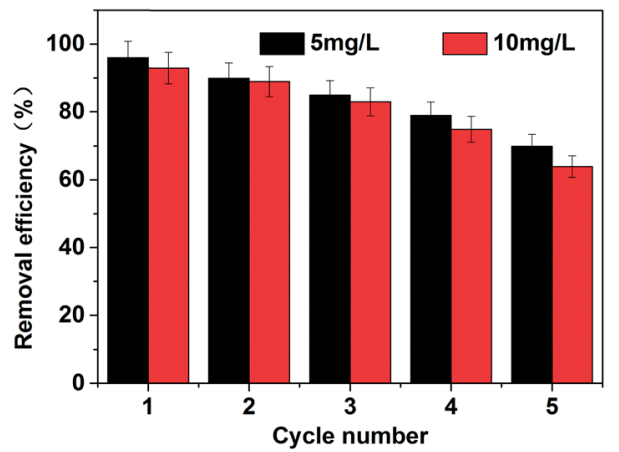

Fig. 5 Removal efficiency of the used Cu-MOFs/PLA films after recovering with acetone washing. 


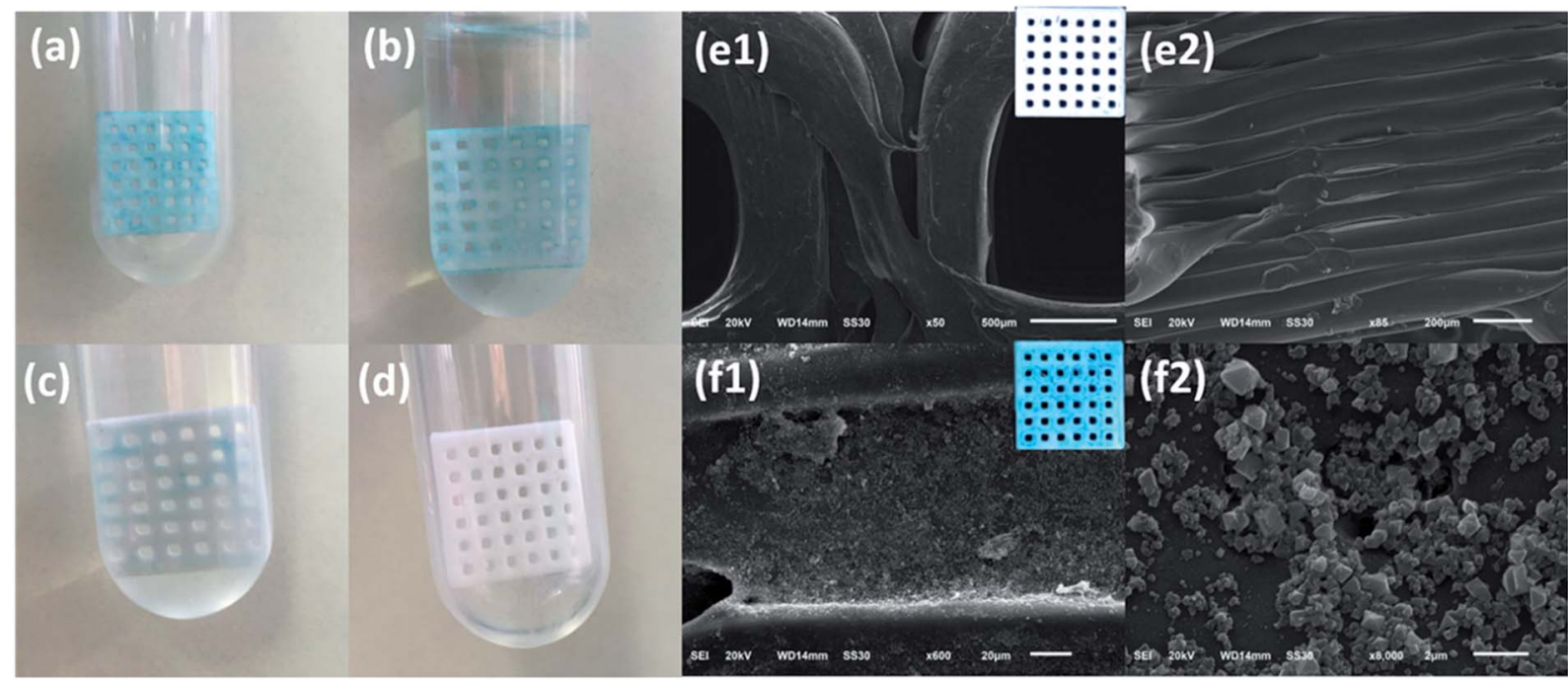

Fig. 6 Images of PLA film regeneration by $\mathrm{HCl}$ treatment (a-d). Used Cu-MOFs/PLA films (a); immersed in $1 \mathrm{M} \mathrm{HCl}$ (b), for $20 \mathrm{~s}$ (c); the PLA skeletons was then obtained after washing with water (d). SEM images of regenerated PLA skeletons (e) and Cu-MOFs/PLA films (f) prepared using in situ growth $\mathrm{Cu}-\mathrm{MOFs}$ on regenerated PLA skeletons.

Table 1 Removal efficiency of Cu-MOFs/PLA films for adsorption of $M G$ in real water samples

\begin{tabular}{lllll}
\hline Sample $^{a}$ & Added & $\begin{array}{l}\text { Concentration } \\
\left(\mathrm{mg} \mathrm{L}^{-1}\right)\end{array}$ & $\begin{array}{l}\text { Found } \\
\left(\mathrm{mg} \mathrm{L}^{-1}\right)\end{array}$ & $\begin{array}{l}\text { Removal efficiency } \\
(\%)\end{array}$ \\
\hline Tap water & MG & 10 & 9.3 & 93 \\
Lake water & MG & 10 & 9.1 & 91 \\
River water & MG & 10 & 9.0 & 90
\end{tabular}

${ }^{a}$ Tap water was collected locally in Wuhan, China; lake water was collected from Shahu Lake in Wuhan; and river water was collected from Yangtze River in Wuhan.

(insert), blue color Cu-MOFs/PLA films were observed. SEM images also showed Cu-MOFs on the PLA skeletons (Fig. 6f), suggesting the successful growth of Cu-MOFs on the used PLA skeletons. The results confirm that the proposed Cu-MOFs/PLA films are renewable, and thus superior for industrial applications for waste-water treatment.

\subsection{Removal of MG from real water samples}

To evaluate the practical applications of prepared Cu-MOFs/PLA films in the removal of MG in real water samples, three types of water, including tap water, lake water, and river water, were collected locally. Filtration was initially performed to remove solid particles, sand, and live organisms, followed by spiking the water with $\mathrm{MG}$ at a concentration of $10 \mathrm{mg} \mathrm{L}^{-1}$. The concentration of MG was measured after immersion of $\mathrm{Cu}$ MOFs/PLA films in samples for $10 \mathrm{~min}$. As shown in Table 1, a high removal efficiency was obtained, with a removal efficiency of $93 \%, 91 \%$, and $90 \%$ for three different samples, respectively. The high efficiencies in adsorption of $\mathrm{MG}$ in real water samples demonstrated that it is feasible to use Cu-MOFs/ PLA films for waste-water treatment, removing MG pollution.

\section{Conclusions}

We report a new approach for the adsorption of organic dyes in waste-water solution by using developed Cu-MOFs/PLA films. These films can easily be prepared by stepwise coating of $\mathrm{Cu}-$ MOFs to the $3 \mathrm{D}$ printed PLA skeleton. The prepared $\mathrm{Cu}-$ MOFs/PLA films were confirmed by SEM images and XRD analysis. Rapid and efficient adsorption of MG was detected with a high removal efficiency of $>90 \%$ within $10 \mathrm{~min}$. The $\mathrm{Cu}-$ MOFs/PLA films were recyclable, and the PLA skeleton could also be regenerated for further use after removal of MG. The regeneration of $\mathrm{Cu}-\mathrm{MOF} / \mathrm{PLA}$ films was confirmed by the in situ coating process. Practical application of this simple, highefficiency and economic Cu-MOFs/PLA film for the adsorption of MG in real water samples was demonstrated. The successful development of this technology is expected to be used for future waste-water treatment in the industry.

\section{Conflicts of interest}

There are no conflicts to declare.

\section{Acknowledgements}

The authors are grateful for financial support from the National Natural Science Foundation of China (Grant No. 51302071, No. 21601076), Wuhan Morning Light Plan of Youth Science and Technology (2017050304010282) and the Australian Research Council (ARC DE170100092).

\section{References}

1 S. Ahad, N. Islam, A. Bashir, S.-u. Rehman and A. H. Pandith, RSC Adv., 2015, 5, 92788-92798. 
2 L. Ai, H. Huang, Z. Chen, X. Wei and J. Jiang, Chem. Eng. J., 2010, 156, 243-249.

3 I. D. Mall, V. C. Srivastava, N. K. Agarwal and I. M. Mishra, Colloids Surf., A, 2005, 264, 17-28.

4 A. Sergi, F. Shemirani, M. Alvand and A. Tajbakhshian, Anal. Methods, 2014, 6, 7744-7751.

5 W. Ngah, W. Saime, N. F. M. Ariff, A. Hashim and M. A. K. M. Hanafiah, Clean: Soil, Air, Water, 2010, 38, 394400.

6 G. Huang, Y. Sun, C. Zhao, Y. Zhao, Z. Song, J. Chen, S. Ma, J. Du and Z. Yin, J. Colloid Interface Sci., 2017, 494, 215-222.

7 M. Hanbali, H. Holail and H. Hammud, Green Chem. Lett. Rev., 2014, 7, 342-358.

8 L. Sun, S. Hu, H. Sun, H. Guo, H. Zhu, M. Liu and H. Sun, RSC Adv., 2015, 5, 11837-11844.

9 J. Fu, Q. Xin, X. Wu, Z. Chen, Y. Yan, S. Liu, M. Wang and Q. Xu, J. Colloid Interface Sci., 2016, 461, 292-304.

10 X. Zhao, S. Liu, Z. Tang, H. Niu, Y. Cai, W. Meng, F. Wu and J. P. Giesy, Sci. Rep., 2015, 5, 11849.

11 M. El-Bouraie, J. Dispersion Sci. Technol., 2015, 36, 12281236.

12 N. Pourreza and S. Elhami, Anal. Chim. Acta, 2007, 596, 6265.

13 M. Cheng, W. Song, W. Ma, C. Chen, J. Zhao, J. Lin and H. Zhu, Appl. Catal., B, 2008, 77, 355-363.

14 W. Fan, W. Gao, C. Zhang, W. W. Tjiu, J. Pan and T. Liu, J. Mater. Chem., 2012, 22, 25108-25115.

15 K. Huang, J. Wang, S. Jiao and H. Zhu, Int. Conf. Mater. Renewable Energy Environ., 2014, 2, 608-612.

16 S. P. Patel, M. Thomas, A. Patel and J. Patel, Int. J. Recent Trends Eng. Res., 2016, 2, 219-229.

17 K. C. de Souza, M. L. P. Antunes, S. J. Couperthwaite, F. T. da Conceição, T. R. de Barros and R. Frost, J. Colloid Interface Sci., 2013, 396, 210-214.

18 H. J. Kumari, P. Krishnamoorthy, T. Arumugam, S. Radhakrishnan and D. Vasudevan, Int. J. Biol. Macromol., 2017, 96, 324-333.

19 A. Omidvar, B. Jaleh and M. Nasrollahzadeh, J. Colloid Interface Sci., 2017, 496, 44-50.

20 A. Keränen, T. Leiviskä, A. Salakka and J. Tanskanen, Desalin. Water Treat., 2015, 53, 2645-2654.

21 A. J. Sami, M. Khalid, S. Iqbal, M. Afzal and A. Shakoori, Pak. J. Zool., 2017, 49, 21-26.

22 M. Yusuf, M. A. Khan, M. Otero, E. Abdullah, M. Hosomi, A. Terada and S. Riya, J. Colloid Interface Sci., 2017, 493, 51-61.

23 H. Han, W. Wei, Z. Jiang, J. Lu, J. Zhu and J. Xie, Colloids Surf., A., 2016, 509, 539-549.

24 K. Sun, L. Li, X. Yu, L. Liu, Q. Meng, F. Wang and R. Zhang, J. Colloid Interface Sci., 2017, 486, 128-135.

25 L. Li, Y. Q. Wu, K. K. Sun, R. Zhang, L. Fan, K. K. Liang and L. B. Mao, Mater. Lett., 2016, 162, 207-210.
26 H. B. Tanh Jeazet, S. Sorribas, J. M. Román-Marín, B. Zornoza, C. Téllez, J. Coronas and C. Janiak, Eur. J. Inorg. Chem., 2016, 2016, 4363-4367.

27 H. A. El Salam, S. Younis, H. Ali and T. Zaki, Microporous Mesoporous Mater., 2017, 241, 210-217.

28 L. Li, X. L. Liu, M. Gao, W. Hong, G. Z. Liu, L. Fan, B. Hu, Q. H. Xia, L. Liu and G. W. Song, J. Mater. Chem. A, 2014, 2, 1795-1801.

29 L. Li, X. L. Liu, H. Y. Geng, B. Hu, G. W. Song and Z. S. Xu, J. Mater. Chem. A, 2013, 1, 10292-10299.

30 O. Shekhah, H. Wang, S. Kowarik, F. Schreiber, M. Paulus, M. Tolan, C. Sternemann, F. Evers, D. Zacher and R. A. Fischer, J. Am. Chem. Soc., 2007, 129, 15118-15119.

31 Y.-Q. Lan, S.-L. Li, X.-L. Wang, K.-Z. Shao, D.-Y. Du, H.-Y. Zang and Z.-M. Su, Inorg. Chem., 2008, 47, 8179-8187. 32 S. Liu, L. Sun, F. Xu, J. Zhang, C. Jiao, F. Li, Z. Li, S. Wang, Z. Wang and X. Jiang, Energy Environ. Sci., 2013, 6, 818-823. 33 Z. Zhang, H. T. H. Nguyen, S. A. Miller, A. M. Ploskonka, J. B. DeCoste and S. M. Cohen, J. Am. Chem. Soc., 2016, 138, 920-925.

34 Z. Shi, L. Li, Y. Xiao, Y. Wang, K. Sun, H. Wang and L. Liu, RSC Adv., 2017, 7, 30904-30910.

35 C. T. Yavuz, J. Mayo, W. Y. William, A. Prakash, J. C. Falkner, S. Yean, L. Cong, H. J. Shipley, A. Kan and M. Tomson, Science, 2006, 314, 964-967.

36 J. Gómez-Pastora, E. Bringas and I. Ortiz, Chem. Eng. J., 2014, 256, 187-204.

37 D. Nagaraju, D. G. Bhagat, R. Banerjee and U. K. Kharul, J. Mater. Chem. A, 2013, 1, 8828-8835.

38 X. Li, L. Zheng, L. Huang, O. Zheng, Z. Lin, L. Guo, B. Qiu and G. Chen, J. Appl. Polym. Sci., 2013, 129, 2857-2864.

39 A. Centrone, Y. Yang, S. Speakman, L. Bromberg, G. C. Rutledge and T. A. Hatton, J. Am. Chem. Soc., 2010, 132, 15687-15691.

40 M. da Silva Pinto, C. A. Sierra-Avila and J. P. Hinestroza, Cellulose, 2012, 19, 1771-1779.

41 S.-M. Lai and Y.-T. Hsieh, J. Macromol. Sci., Part B: Phys., 2016, 55, 211-228.

42 K. Oksman, M. Skrifvars and J.-F. Selin, Compos. Sci. Technol., 2003, 63, 1317-1324.

43 Ö. C. Önder, E. Yilgör and I. Yilgör, Polymer, 2016, 107, 240248.

44 C. Esposito Corcione, F. Gervaso, F. Scalera, F. Montagna, A. Sannino and A. Maffezzoli, J. Appl. Polym. Sci., 2017, 37, 741-746.

45 K. J. Christiyan, U. Chandrasekhar and K. Venkateswarlu, J. Inst. Eng., 2016, 1-5.

46 K. M. Nampoothiri, N. R. Nair and R. P. John, Bioresour. Technol., 2010, 101, 8493-8501.

47 F. Ke, L.-G. Qiu, Y.-P. Yuan, F.-M. Peng, X. Jiang, A.-J. Xie, Y.-H. Shen and J.-F. Zhu, J. Hazard. Mater., 2011, 196, 36-43. 